\title{
Geotecnologias Aplicada ao Planejamento Urbano: Um Estudo de Caso Sobre a Acessibilidade na Comunidade Alto Bela Vista, Pacoti - Ceará.
}

\author{
Geotechnology Applied to Urban Planning: A Case Study About Accessibility in the \\ Community Alto Bela Vista, Pacoti - Ceará.
}

VALDIVINO $^{1}$, L. F.X.; MATOS $^{2}$, L. S.; OLIVEIRA ${ }^{3}$, F. L.S;

luis.felipe@aluno.uece.br

\begin{abstract}
Resumo
O presente trabalho tem por objetivo principal utilizar-se das técnicas de geoprocessamento aplicado na identificação de áreas com acessibilidade comprometida na Comunidade Alto Bela Vista, no município de Pacoti, Ceará. A pesquisa foi operacionalizada através de levantamentos e revisão bibliográfica e atividades de campo. Além da utilização do Software Google Earth Pro para identificação das residências. Visando nortear a população e as autoridades acerca das condições de acessibilidade na comunidade Alto Bela Vista e as principais barreiras urbanísticas enfrentadas pela população, em especial as pertencentes ao grupo de pessoas mais vulneráveis como crianças menores de dez anos, idosos com mais de sessenta anos e pessoas com dificuldade de locomoção, foi apresentado um panorama atual das condições de acessibilidade na comunidade, sendo quantificado o número de moradores que são afetados pela precária infraestrutura urbana do bairro. Além disso, constatou-se que na comunidade Alto Bela Vista verificou-se que a precária situação da infraestrutura urbana não vêm atendendo de forma hábil aos moradores, em especial os mais vulneráveis, que representam 13,6\% da população residente na comunidade. Por fim, vale ressaltar que o presente estudo encontra-se em estágio inicial, evidenciando-se a necessidade de prosseguir com as análises pertinentes ao planejamento urbano atrelado as geotecnologias.
\end{abstract}

Palavras-chave:geoprocessamento, planejamento urbano, acessibilidade.

\begin{abstract}
This study's main objective is to use geoprocessing techniques applied in identifying areas with impaired accessibility in the Community Alto Bela Vista, in the municipality of Pacoti, Ceará. The research was implemented through surveys and bibliographic and field activities review. In addition to using Google Earth Pro software for identification of households. Aiming to guide the population and the authorities about the accessibility conditions in Alto Bela Vista community and key urban barriers faced by the population, especially those belonging to the group of more vulnerable people such as children under ten years old, people over sixty years and people with limited mobility, a current overview of accessibility conditions in the community was presented, and quantified the number of residents who are affected by poor urban infrastructure of the neighborhood. In addition, it was found that in Alto Bela Vista community found that the precarious situation of urban infrastructure have not given skillfully residents, especially the most vulnerable, representing $13.6 \%$ of the resident population in the community. Finally, it is noteworthy that this study is in its early stages, demonstrating the need to continue with the relevant analysis to urban planning linked geotechnologies.
\end{abstract}

Keywords: GIS, urban planning, accessibility

\section{INTRODUÇÃO}

De acordo com (Nascimento, Lima e Santos, 2009), o processo de urbanização brasileira adicionará aproximadamente 54 milhões de pessoas as cidades de todo o país, elevando a população para cerca de mais de 200 milhões de habitantes, desencadeando um significativo potencial de transferência da população do campo para as cidades.

${ }^{1}$ Luis Felipe Xavier Valdivino, Centro de Ciencia e Tecnologia, Universidade Estadual do Ceará, Fortaleza, CE, Brasil.

${ }^{2}$ Luciola Silva de Matos, Centro de Ciencia e Tecnologia, Universidade Estadual do Ceará, Fortaleza, CE, Brasil.

${ }^{3}$ Francisca Leiliane Sousa de Oliveira, Laboratório de Geoprocessmaneot e Estudos Aplicados, Universidade Estadual do Ceará, Fortaleza, CE, Brasil. 
Devido a esse acelerado crescimento dos centros urbanos, as populações passaram a ocupar áreas improprias para moradia, configurando o surgimento de habitações em áreas de risco.

Em Pacoti, o processo de ocupação da sede municipal foi caracterizado pelo uso intensivo do solo com edificações, infraestruturas viárias, localizadas conforme as características do meio físico e vias de acesso, ou seja, em vertentes íngremes ou em fundos de vale.

A concentração da ocupação às margens do rio Pacoti e seus afluentes, é uma das áreas de principal preocupação, além das vertentes íngremes, que vem contribuindo para a formação de habitações desprovidas de infraestrutura, dificultando o fluxo das pessoas com deficiências ou dificuldade de locomoção nos espaços urbanos, restringindo o exercício da cidadania e prejudicando o convívio social, sendo assim caracterizado como barreiras urbanísticas. (Goiais, 2013)

Além disso, boa parte da acessibilidade dos bairros em sua área urbana, apresentam declives acentuados e precária infraestrutura de calçamentos e calçadas, dificultando a locomoção principalmente de indivíduos pertencentes ao grupo de pessoais mais vulneráveis, como idosos, crianças e pessoas com deficiência física.

Visando otimizar as análises sobre planejamento urbano e a acessibilidade na sede municipal de Pacoti, o presente trabalho adotou o amparo do geoprocessamento para suas análises e representação de dados espaciais.

\section{METODOLOGIA}

Para o desenvolvimento da presente pesquisa foram realizados levantamento de dados bibliográficos, cartográficos e documental. Posteriormente foi realizado atividade de campo na área em estudo, com a realização de entrevistas e aplicação de questionários com moradores da comunidade Alto Bela Vista.

A operacionalização da pesquisa foi constituída na produção de tabelas, gráficos e fontes documentais do município, além da utilização do Google Earth Pro para identificação das residências onde habitam os indivíduos pertencentes ao grupo dos mais vulneráveis à ausência de infraestrutura urbana.

Com a realização do trabalho de campo e as entrevistas, pode-se quantificar o número de habitantes pertencentes ao grupo de indivíduos mais vulneráveis, sendo esses as crianças menores de 10 anos, idosos com mais de 60 anos e pessoas com deficiência física. 
$\mathrm{Na}$ etapa de pré-campo foram identificadas todas as residências da Comunidade Alto Bela Vista, e posteriormente, em gabinete, com os dados obtidos pode-se quantificar o numero de indivíduos que fazem parte do grupo de pessoas mais vulneráveis.

O uso de aplicações do Sistema de Informação geográfica - SIG no planejamento urbano são entendidas a partir de IPT (2007) expressos no Quadro 01:

Quadro 01. Aplicações do SIG no planejamento urbano. Fonte: IPT (2007)

\begin{tabular}{|c|c|}
\hline & $\begin{array}{l}\text { Principais aplicações do SIG no planejamento } \\
\text { Urbano. }\end{array}$ \\
\hline 1. & $\begin{array}{l}\text { Ordenamento e gestão do território - permite a constituição de uma base } \\
\text { cartográfica georeferenciada que servirá às demais aplicações setoriais. } \\
\text { Trata-se de construir uma base de dados informatizada que reproduza a } \\
\text { configuração do território do município, identificando logradouros, lotes e } \\
\text { glebas, edificações, redes de infraestrutura, propriedades rurais, estradas e } \\
\text { acidentes geográficos. }\end{array}$ \\
\hline 2 & $\begin{array}{l}\text { Otimização de arrecadação - a atualização da base carto- gráfica do } \\
\text { município fornece informações para a revisão da planta genérica de valores. } \\
\text { Localização de equipamentos e serviços públicos - inclusão de informações } \\
\text { socioeconômicas e sobre equipamentos públicos, o que torna possível a } \\
\text { identificação das áreas com maior nível de carência e os melhores locais } \\
\text { para instalação de equipamentos e serviços públicos. }\end{array}$ \\
\hline 3. & $\begin{array}{l}\text { Identificação de público-alvo de políticas públicas incorporar dados } \\
\text { socioeconômicos, onde se pode identificar o público alvo para aplicação de } \\
\text { programas públicos. Gestão ambiental - monitorar áreas com maior } \\
\text { necessidade de proteção ambiental, acompanhar a evolução da poluição da } \\
\text { água e do ar, níveis de erosão do solo, disposição irregular de resíduos e } \\
\text { para o gerenciamento dos serviços de limpeza pública. }\end{array}$ \\
\hline
\end{tabular}




\begin{tabular}{|l|l|}
\hline 4. & \begin{tabular}{l} 
É possível a elaboração de mapas de riscos que auxiliam na elaboração de \\
rotas de fuga. Gerenciamento do sistema de transportes - realizar estudos de \\
demanda do transporte coletivo ou de carregamento de vias, identificar \\
pontos críticos de acidentes e vias com mais necessidade de manutenção. \\
Comunicação com os cidadãos - pode-se incorporar a ela informações que \\
permitam identificar necessidades e oportunidades de contato com os \\
cidadãos. \\
\hline Gestão da frota municipal - é possível obter informações sobre os tipos de \\
usos da frota municipal, conhecendo os trajetos mais comuns e sua \\
intensidade. Estas informações possibilitarão a definição de roteiros \\
otimizados para a frota municipal, gerando economia de tempo, combustível \\
e uso de veículos.
\end{tabular} \\
\hline
\end{tabular}

\section{RESULTADOS E DISCUSSÃO}

A partir das análises realizadas pela aquisição de imagens do Google Earth percebeu-se que o município de Pacoti teve uma expansão significante da sua malha urbana, tendo como base dados da mancha urbana do ano de 2010 adquiridas no Instituto de Pesquisa e Estratégia Econômica do Ceará - IPECE, comparando com imagens disponíveis no Google Earth do ano de 2016.

A ocupação da área urbana de Pacoti se deu de forma desordenada, sem a devida intervenção de planejamento territorial por parte do poder publico. Fato que acarretou na ocupação de áreas localizadas principalmente as margens do rio Pacoti e nas vertentes íngremes localizadas na área urbana de Pacoti.

$\mathrm{Na}$ comunidade Alto Bela Vista, localizada na sede municipal de Pacoti, apresenta construções que ocupam sobretudo zonas de instabilidade na base e no topo da encosta, favorecendo assim uma maior possibilidade da ocorrência de deslizamento de terra.

Tais fatores atrelados as exíguas ações de planejamento urbano, acabaram contribuindo para a existência de áreas precárias no que diz respeito a infraestrutura urbana do bairro, além de contribuir na existência de área de grande risco de deslizamento.

A ausência de planejamento urbano e a falta de intervenção dos gestores públicos na infraestrutura urbana da sede municipal levaram os moradores a criarem seus próprios meios de acessos as suas residências, muitos desses de forma improvisada e precária, dificultando o acesso principalmente de crianças, idosos e deficientes físicos a esses locais. Se constituindo verdadeiras barreiras urbanísticas, principalmente para os idosos, crianças e deficientes. 
Com os dados coletados em campo, pode-se verificar a existência de um número considerável de moradores na comunidade Alto bela Vista pertencentes ao grupo de indivíduos mais vulneráveis, que enfrentam diariamente dificuldades de locomoção devido à ausência de acessibilidade adequada a esse grupo, como também ao restante da população. No gráfico da figura 01 podemos observar esses dados:

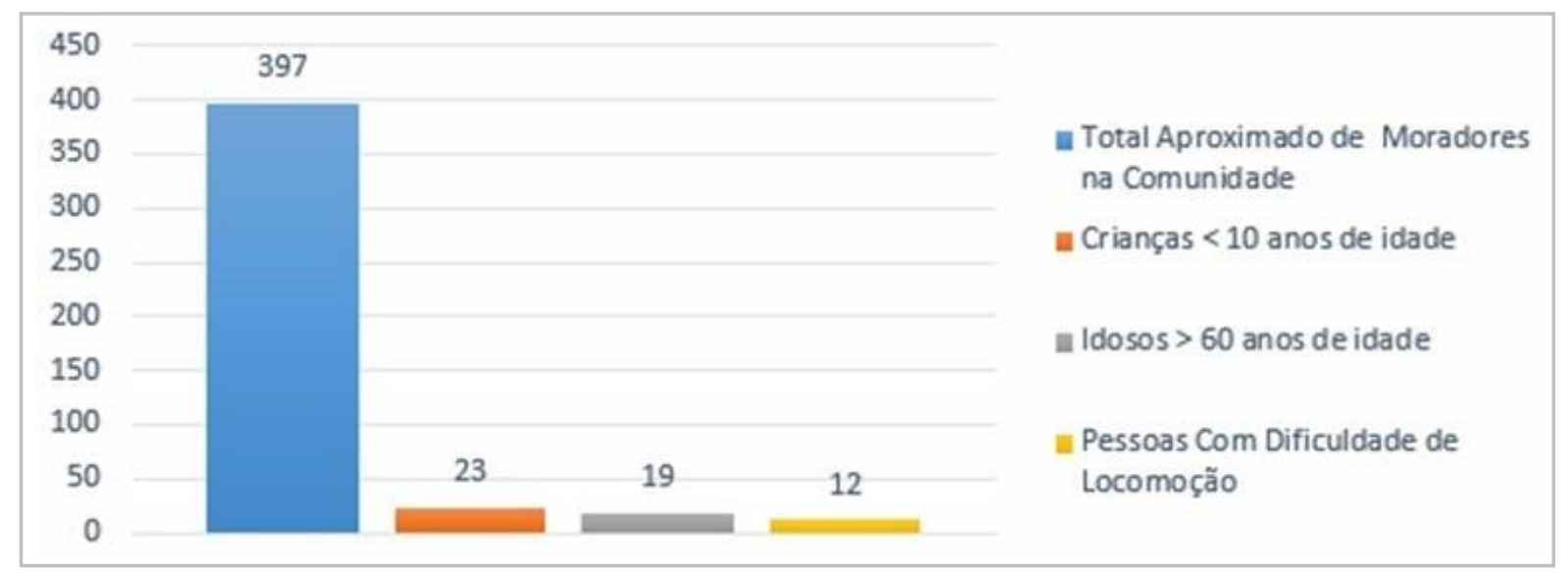

Figura 1: Número de pessoas mais vulneráveis que moram na comunidade alto bela vista, município de Pacoti. Fonte: Autores. (2016)

Como apresentado no gráfico acima, dos 397 habitantes da Comunidade Alto Bela Vista, foi quantificados um total de 54 moradores pertencentes ao grupo dos mais vulneráveis, sendo as crianças de até 10 anos, idosos com mais de 60 anos e pessoas com limitações de locomoção, representando um total de $13,6 \%$ de pessoas mais vulneráveis em cenário de risco.

A ausência de infraestrutura urbana que promova a locomoção propicia a esses grupos limita as caches de evacuação dessa população em cenários de risco, como um deslizamento de terra que possa vim a ocorrer na Comunidade Alto Bela Vista. Além do acesso precário que dificultaria a chegada de ambulâncias, corpo de bombeiros e etc.

Existem diversos acessos na comunidade Alto Bela Vista feitos de forma improvisada pela própria população, sem nenhum suporte da prefeitura. Contudo, esses acessos não apresentam nenhuma rampa ou corrimão de acesso para idosos ou deficientes físicos.

A comunidade possui apenas dois acessos oficiais, sendo que apenas um deles permite a passagem de veículos automotivos, mas que se encontra em significativo grau de deterioração. $\mathrm{Na}$ figura 2 abaixo, podemos observar o momento em que uma ambulância subia pelo único acesso que permite a passagem de veículos, sendo uma rua estreita e íngreme, dificultando ainda mais a chegada do serviço. 


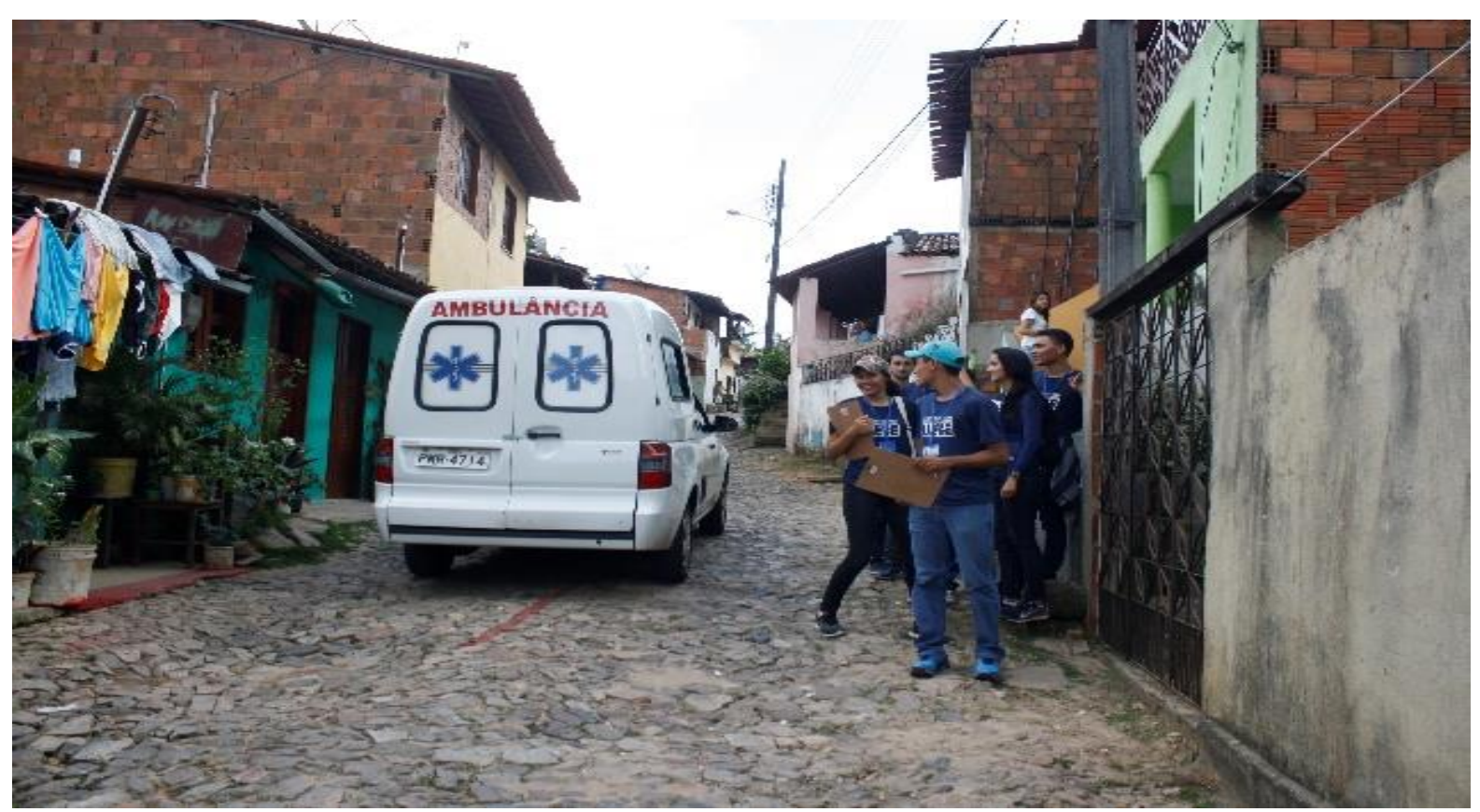

Figura 2: Ambulância no acesso oficial que permite o trafego de veículos na comunidade Alto Bela Vista.

Fonte: Autores (2016)

Outro fator que torna a promoção da acessibilidade na Comunidade Alto Bela Vista uma atividade complexa são as escadarias existentes no segundo acesso oficial na comunidade, permitindo apenas a circulação de pedestres. As escadarias não seguem um padrão, além de não apresentarem corrimões ou rampas para a locomoção de pessoas com limitações de locomoção (Vide figura 3 e 4 ).

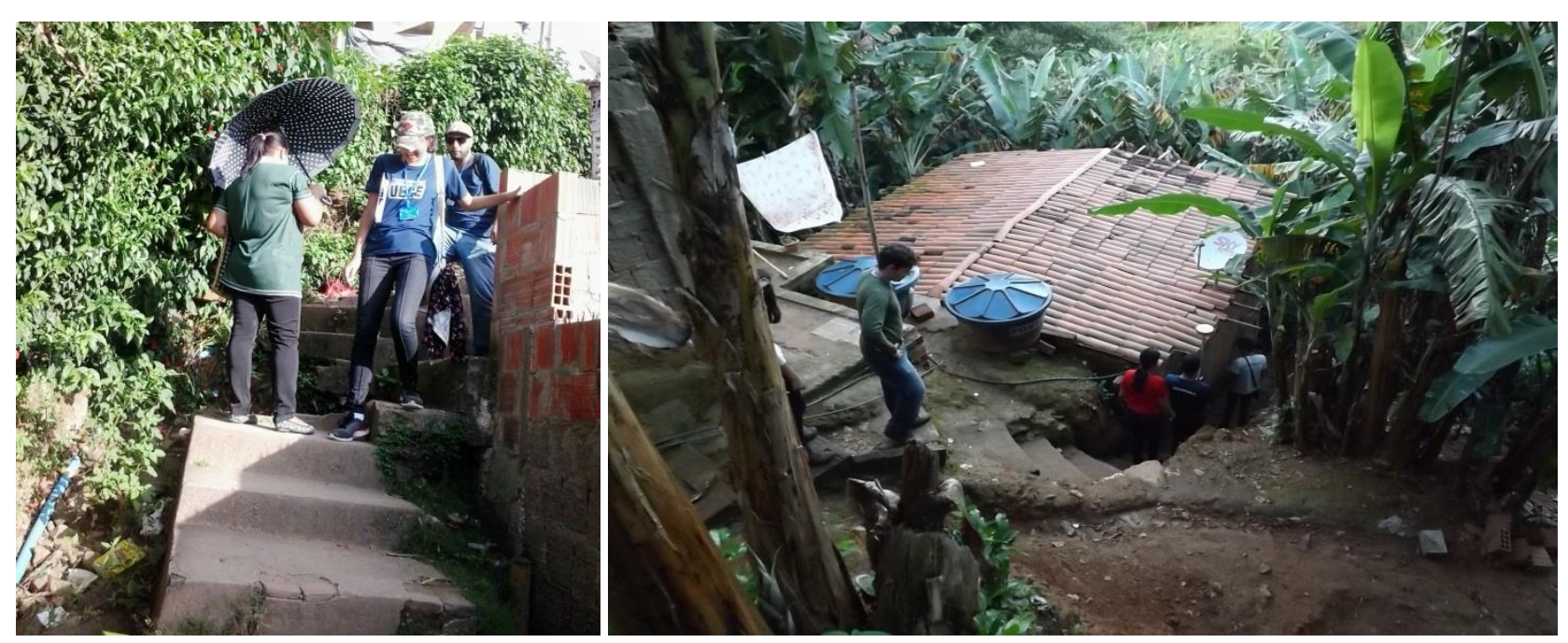

Figuras 3 e 4 - Escadaria de acesso oficial (administrado pela prefeitura) à comunidade Alto Bela Vista e escada emprovisada pelos morados para acesso a residências. Fonte: Autores (2016). 
Diante dos trabalhos de campos com registros fotográficos pode-se verificar as principais barreiras urbanísticas e os aspectos ligados a ausência de acessibilidade na comunidade Alto Bela Vista, fatores que dificultam ainda mais a vida dos moradores diariamente, além do acesso de veículos automotivos, e consequentemente a chegada de serviços públicos como: ambulâncias, corpo de bombeiros dentre outros.

A partir dos dados obtidos em campo com a aplicação dos questionários, pode-se realizar a localização dos domicílios onde residem as pessoas pertencentes ao grupo dos indivíduos mais vulneráveis à falta de acessibilidade localizada na comunidade Alto Bela Vista.

Posteriormente, utilizou-se do Google Earth Pro para identificar todos os domicílios da comunidade Alto Bela Vista, sendo marcados pontos definidos no pré-campo e posteriormente verificados in loco os domicílios que haviam moradores pertencente ao grupo de indivíduos mais vulneráveis. Cujo resultado é exposto na figura 04 abaixo:

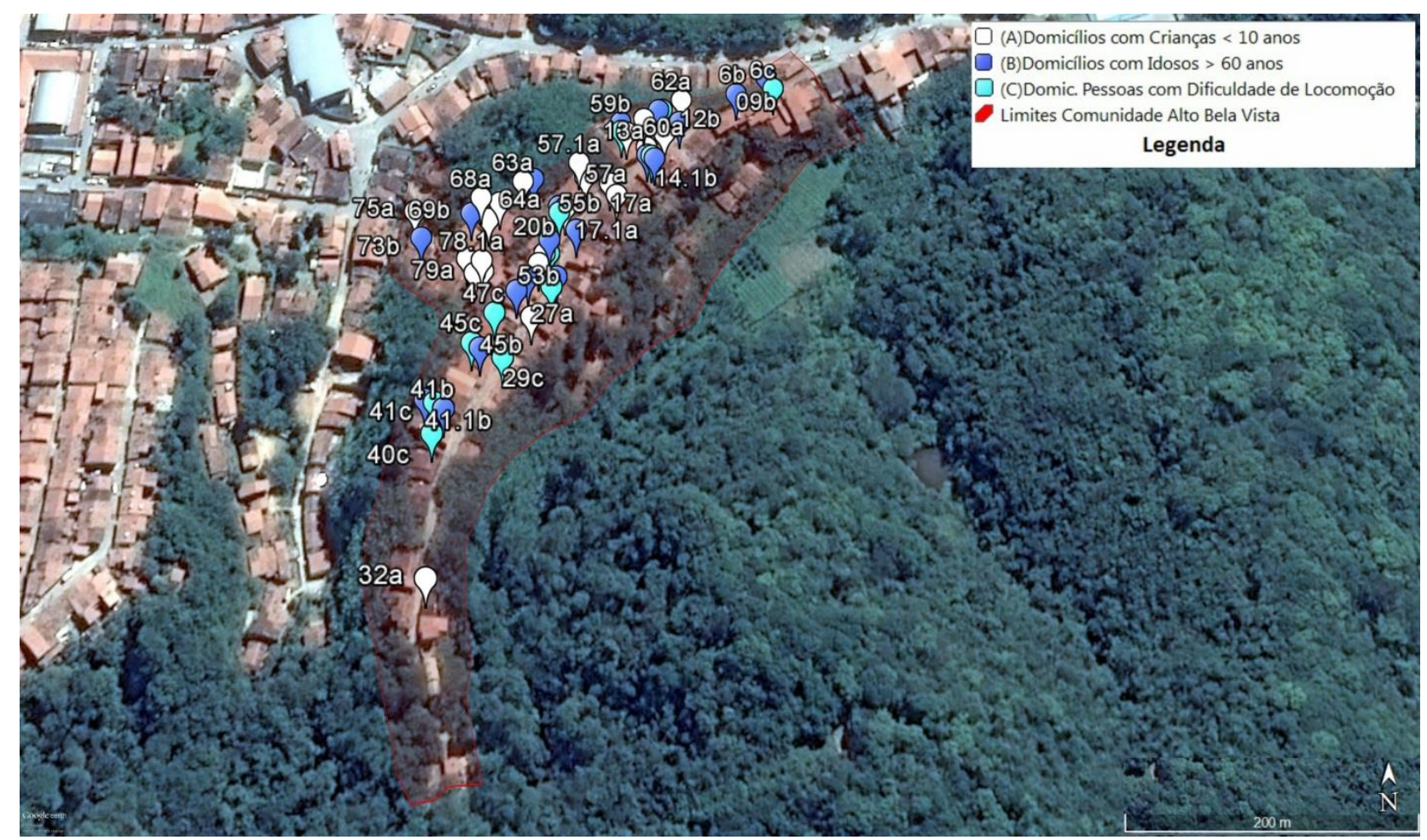

Figura 05: Imagem do Google Earth a localização dos domicílios com crianças, idosos e pessoas com dificuldade de locomoção. Elaboração: Autores. (2016) Imagem: Google Earth Pro.

A localização dos desses domicílios a partir dos pontos coletados em campo irá subsidiar na identificação e chegada de serviços de socorro, em cenário de eventos adversos das pessoas que integram o grupo das mais vulneráveis. Além de auxiliar na elaboração de intervenções de promoção a acessibilidade. 


\section{CONSIDERAÇÕES FINAIS}

No presente estudo de caso, pode-se identificar os grupos mais vulneráveis na comunidade alto bela vista, observando as principais barreiras urbanistas enfrentadas pelos moradores da área. Sendo possível diagnosticar o número de indivíduos atingidos pela ausência ou precária infraestrutura física de acesso.

Nota-se ainda que há muito a ser feito no que diz respeito ao planejamento urbano nas áreas de expansão da sede municipal de Pacoti. Tendo em vista o acentuado adensamento populacional resultando na formação de assentamentos precários em termos de infraestrutura, e, portanto, mais vulneráveis.

Além disso, é importante salientar que o presente estudo ainda encontra-se em estágio inicial, sendo pertinência a continuidade nas analises, com auxilio das geotecnologias como ferramenta potencializadora nos estudos geográficos, e atreladas ao planejamento urbano e na formulação de politicas publica de promoção à acessibilidade, em especial nas áreas de risco da sede municipal de Pacoti.

\section{REFERÊNCIAS}

CASTRO, Antonio Luiz Coimbra de. Manual de planejamento em defesa civil. Brasília: Ministério da Integração Nacional, Secretaria de Defesa Civil, 1999.

GOIAS, Ministério Público do Estado de Goiás Disponível em: http://www.mpgo.mp.br/portal/arquivos/2013/08/22/14_41_39_381_Cartilha_viva_acessibilidade2 1.pdf. Acessado em 08 de agosto de 2016.

IBGEcidades,2010.Disponívelemhttp://cidades.ibge.gov.br/xtras/perfil.php?lang=\&amp;codmun=2 30140\&amp;search=ceara|pacoti. Acessado em 10 de agosto de 2016.

LEITE, M. E; .LEITE, M. R. ; PEREIRA, D. M. ; BRITO, J. L. S. . Sensoriamento remoto aplicado ao monitoramento dos assentamentos urbanos ilegais. In: Simpósio Brasileiro de Sensoriamento Remoto, 2013, Foz do Iguaçu. XVI Simpósio Brasileiro de Sensoriamento Remoto. INPE,2013.v.1.p.1-15.

Instituto de Pesquisas Tecnológicas - IPT. Mapeamento de Riscos em Encostas e Margem de Rios / Celso Santos Carvalho, Eduardo Soares de Macedo e Agostinho Tadashi Ogura, organizadores - Brasília: Ministério das Cidades; Instituto de Pesquisas Tecnológicas - IPT, 2007. MOURA, Ana Clara M.; XAVIER-DA-SILVA, J. . Geoprocessamento aplicado à caracterização e planejamento urbano de Ouro Preto - MG. In: XAVIER-DA-SILVA, Jorge; ZAIDAN, Ricardo. (Org.). Geoprocessamento e Análise Ambiental - Aplicações. 1ed. Rio de Janeiro: Bertrand 
Brasil,2004,v.1,p.217-258.

NASCIMENTO, Vânia Maria Lima Carneiro ; LIMA, E. R. V. ; SANTOS, Celso Augusto Guimarães . Sig Na Avaliação De Áreas Para Ocupação Urbana De João Pessoa/Pb. Ambiente Construído(Online),v.9,p.107-123,2009.

OLIVEIRA, F. L. S.; CRUZ, M. L. B. Mapeamento e Análise dos Recursos Naturais do Município De Pacoti, Ceará, Brasil. In: XIV SIMPÓSIO BRASILEIRO DE GEOGRAFIA FÍSICA APLICADA, 2011, Dourados. Ipê Multimídia, 2011., 2011. v. v. 1.

Universidade Federal de Santa Catarina. Centro Universitário de Estudos e Pesquisas sobre Desastres. Assessoria técnica e avaliação de municípios: resultados da avaliação dos perfis municipais / Centro Universitário de Estudos e Pesquisas sobre Desastres. - Florianópolis: CEPED UFSC, 2015.

Universidade Federal de Santa Catarina. Centro Universitário de Estudos e Pesquisas sobre Desastres. Mobilização comunitária para a redução de riscos de desastres/ Centro Universitário de Estudos e Pesquisas sobre Desastres - Florianópolis: CEPED UFSC, 2015. 86 p.

Recebido em: 14/08/2016

Aceito para publicação em: 01/10/2016 